\section{Outbreak of West Nile Virus Infection in Greece, 2010}

Kostas Danis, Anna Papa,

George Theocharopoulos, Georgios Dougas, Maria Athanasiou, Marios Detsis, Agoritsa Baka, Theodoros Lytras, Kassiani Mellou, Stefanos Bonovas, and Takis Panagiotopoulos

During 2010, an outbreak of West Nile virus infection occurred in Greece. A total of 197 patients with neuroinvasive disease were reported, of whom 33 (17\%) died. Advanced age and a history of heart disease were independently associated with death, emphasizing the need for prevention of this infection in persons with these risk factors.

A $\mathrm{n}$ outbreak of West Nile virus (WNV) infection occurred in Central Macedonia in northern Greece in the summer of 2010. The first cases were diagnosed and reported to the Hellenic Centre for Disease Control and Prevention (HCDCP) on August 5, 2010 (1). WNV lineage 2 sequences were later obtained from 3 pools of Culex pipiens mosquitoes trapped at 2 sites where cases of West Nile neuroinvasive disease (WNND) had occurred (2).

Human cases of WNV disease had not been previously reported in Greece. Serosurveys in the early 1960s, 1980s, and 2007 identified WNV antibodies in $\approx 1 \%$ of the population, suggesting that $\mathrm{WNV}$, or a related flavivirus, was circulating in Greece (3-5). In contrast, during 2005-2007, a total of 9,590 blood donors were tested by WNV nucleic acid amplification assay and results were negative (6).

\section{The Study}

After the outbreak alert was issued in early August 2010, physicians in Greece were asked to report all cases of WNV infection to HCDCP, according to the current European Union case definition (1). Only deaths that occurred during hospitalization were attributed to WNV infection. Statistical methods are described in the online Technical Appendix (www.cdc.gov/EID/content/17/10/110525-Techapp.pdf).

Author affiliations: Hellenic Centre for Disease Control and Prevention, Athens, Greece (K. Danis, G. Theocharopoulos, G. Dougas, M. Athanasiou, M. Detsis, A. Baka, T. Lytras, K. Mellou, S. Bonovas, T. Panagiotopoulos); Aristotle University of Thessaloniki, Thessaloniki, Greece (A. Papa); and National School of Public Health, Athens (T. Panagiotopoulos)

DOI: http://dx.doi.org/10.3201/eid1710.110525
Serum and cerebrospinal fluid specimens were tested for immunoglobulin (Ig) $\mathrm{M}$ and IgG against WNV by using an ELISA (WNV IgM capture DxSelect and WNV IgG DxSelect; Focus Diagnostics Inc., Cypress, CA, USA). Titers $>640$ were obtained by microneutralization assay against WNV in 14/14 patients who were positive for WNV. Although tick-borne encephalitis virus (TBEV) and dengue virus (DENV) are not prevalent in Greece, specimens were also tested for TBEV and DENV by ELISA (TBE/ FSME IgM and TBE/FSME IgG; IBL International Gmbh, Hamburg, Germany) and Dengue Virus IgM Capture Dx Select and IgG Dx Select (Focus Diagnostics Inc.). All specimens were negative for TBEV, and cross-reactivity was seen with DENV, mainly for IgM (7).

Overall, 262 patients with WNV infection were reported to HCDCP. Of these patients, 197 (75\%) had neuroinvasive disease (encephalitis, meningitis, or acute flaccid paralysis), and 65 (25\%) had WNV fever. This study focused on patients with WNND, who were identified and reported more consistently because of disease severity.

Patient disease onset occurred within a 14-week interval during July 6-October 5 , and the outbreak peaked in mid August (Figure 1). Most (94\%) patients with WNND were reported from the 7 districts of Central Macedonia (Figure 2), and the epicenter of the outbreak was in Pella and Imathia Districts.

Characteristics of patients with WNND are shown in Table 1. Median age of patients with neuroinvasive disease was 72 years (range $12-88$ years). The attack rate for WNND increased significantly $(\mathrm{p}=0.006)$ with age (Table 1). The incidence of WNND in older persons $(\geq 80$ years of age) was $\approx 50 \times$ higher than that among the youngest age

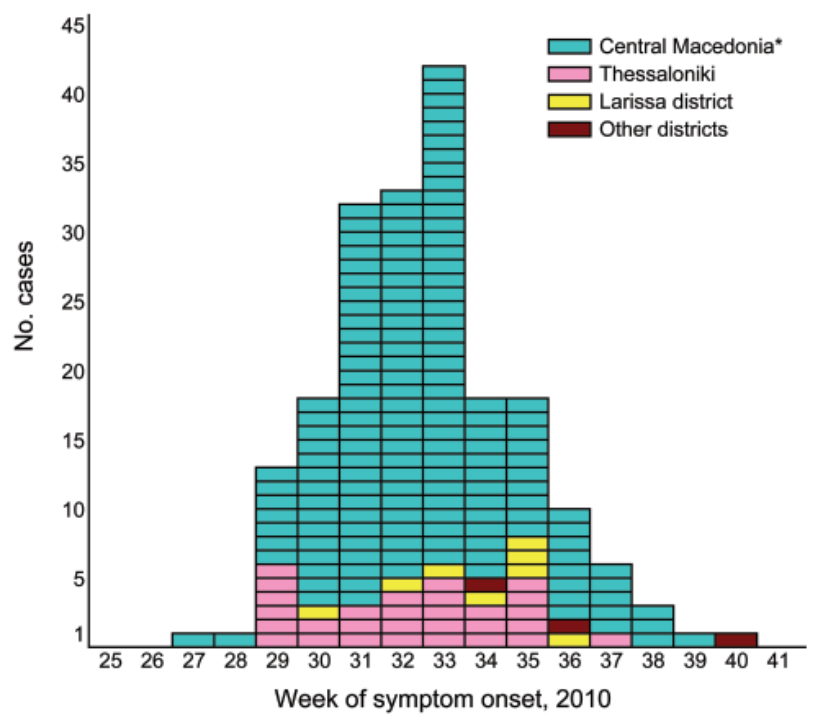

Figure 1. Reported cases $(n=197)$ of West Nile neuroinvasive disease, by week of symptom onset, Greece, July 6-October 5, 2010. *Excluding Thessaloniki. 


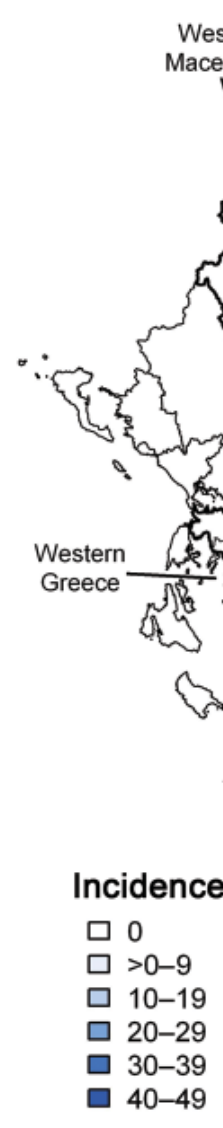

Figure 2. Incidence per 100,000 population of 197 reported cases of West Nile neuroinvasive disease, by township of residence, Greece, July-October 2010. Districts with $\geq 1$ reported neuroinvasive cases were divided into townships. Dark black lines indicate borders of Central Macedonia (north) and Thessalia (south).

group $(<20$ years of age). Persons living in rural areas were $2 \times$ as likely to show development of WNND than persons living in urban areas (Table 1).

Encephalitis/meningoencephalitis (168 patients, 85\%) was the most prominent clinical syndrome among patients with WNND, followed by meningitis $(23,12 \%)$. In addition, $10(5 \%)$ patients with acute flaccid paralysis were reported, 6 (3\%) of whom did not have meningitis or encephalitis.

A large proportion (74\%) of patients with WNND had $\geq 1$ underlying chronic medical condition; the most common were hypertension (39\%), heart disease (24\%), diabetes mellitus (24\%), and immunosuppression (10\%). Patients with WNND were $2 \times$ more likely (odds ratio 2.16 , $95 \%$ confidence interval $1.15-4.04)$ than patients without WNND to have underlying conditions.

Thirty-five patients died during hospitalization (33 had WNND), indicating an overall case-fatality rate of $17 \%$ among persons with WNND. Median age of WNND patients who died was 78 years (range $49-87$ years). The case-fatality rate increased substantially $(\mathrm{p}<0.001)$ with age (Table 2). Median interval from WNV disease onset to death was 13 days (range 3-90 days). In 15 (45\%) patients with WNND who died, the interval between disease onset and death exceeded 2 weeks.
WNND patients with $\geq 1$ underlying disease were $5 \times$ more likely to have died than patients without underlying conditions. Those patients who had heart disease or a stroke were $\approx 2.5 \times$ more likely to have died than patients without these conditions. However, only older age and heart disease were independent predictors of death in the final binomial regression model (Table 2). Supplementary results are shown in the online Technical Appendix.

\section{Conclusions}

Human cases of WNV infection were detected in several European and Mediterranean countries in 2010, indicating an increased intensity of viral circulation (8). Clinical cases of WNV infection in humans or animals had not been previously reported in Greece. The present outbreak was the largest in Europe since 1996, when a large outbreak was observed in Romania (9). The outbreak was located in Central Macedonia, which contains $90 \%$ of the rice paddies and $70 \%$ of the wetland areas in Greece and provides a favorable environment for reproduction of mosquito vectors (8). The region also hosts one of the largest populations of migratory birds in Greece. Meteorologic data for the area indicate that 2010 was warmer than previous years and unusually wet (8). 


\section{DISPATCHES}

Table 1. Characteristics of 197 patients with West Nile neuroinvasive disease. Greece, July-October 2010*

\begin{tabular}{|c|c|c|c|}
\hline Characteristic & No. patients & Incidence per 100,000 population & Risk ratio $(95 \% \mathrm{Cl})$ \\
\hline \multicolumn{4}{|l|}{ Age group, y } \\
\hline$<20$ & 4 & 0.18 & Reference \\
\hline $20-29$ & 3 & 0.20 & $1.08(0.24-4.84)$ \\
\hline $30-39$ & 6 & 0.34 & $1.87(0.53-6.63)$ \\
\hline $40-49$ & 9 & 0.55 & $3.03(0.93-9.82)$ \\
\hline $50-59$ & 18 & 1.27 & $6.93(2.35-20.49)$ \\
\hline $60-69$ & 29 & 2.44 & $13.31(4.68-37.84)$ \\
\hline $70-79$ & 85 & 8.01 & $43.74(16.05-119.2)$ \\
\hline$\geq 80$ & 43 & 9.63 & $52.62(18.89-146.6)$ \\
\hline \multicolumn{4}{|l|}{ Sex } \\
\hline $\mathrm{F}$ & 88 & 1.59 & Reference \\
\hline M & 109 & 1.97 & $1.26(0.95-1.67)$ \\
\hline \multicolumn{4}{|l|}{ Place of residence } \\
\hline Urban & 110 & 1.38 & Reference \\
\hline Rural & 87 & 2.92 & $2.12(1.60-2.80)$ \\
\hline \multicolumn{4}{|l|}{ Districts in Central Macedonia } \\
\hline Chalkidiki & 4 & 3.99 & $0.76(0.28-2.09)$ \\
\hline Thessaloniki & 60 & 5.27 & Reference \\
\hline Pieria & 9 & 7.02 & $1.33(0.66-2.69)$ \\
\hline Serres & 21 & 11.15 & $2.12(1.29-3.48)$ \\
\hline Kilkis & 12 & 13.92 & $2.64(1.42-4.91)$ \\
\hline Imathia & 39 & 27.06 & $5.14(3.43-7.69)$ \\
\hline Pella & 41 & 28.26 & $5.37(3.61-7.98)$ \\
\hline Total & 186 & 15.00 & NA \\
\hline \multicolumn{4}{|l|}{ Other districts (region) } \\
\hline Etoloakarnania (western Greece) & 1 & 0.46 & $0.09(0.01-0.63)$ \\
\hline Kozani (Western Macedonia) & 1 & 0.65 & $0.12(0.02-0.89)$ \\
\hline Kavala (Eastern Macedonia) & 1 & 0.71 & $0.14(0.02-0.98)$ \\
\hline Larissa (Thessalia) & 8 & 2.80 & $0.53(0.25-1.11)$ \\
\hline Total in Greece & 197 & 1.76 & NA \\
\hline
\end{tabular}

The overall case-fatality rate among patients with WNND (17\%) was higher in Greece than that in other countries (9-11). The reasons for this finding are not clear. Many factors may have played a role in differences in the fatality rate. These factors include diagnosis and surveillance bias for more severe cases, virus strain, host susceptibility, age structure of the population, and underlying conditions.

Recent studies on WNV lineage 2 suggested that this virus may be underestimated as a cause of neuroinvasive disease $(2,12)$. WNV linage 2 isolated from $C x$. pipiens mosquitoes in the affected areas during this outbreak had a nucleotide genetic similarity of $99.6 \%$ with the goshawk Hungary 2004 strain (12). However, few severe cases of WNV infection were reported in Hungary. Experimental studies would verify whether the amino acid substitution H249P detected in the Greek strain, which is a suspected virulence marker in lineage 1 strains, is associated with increased virulence (12).

Advanced age and heart disease were found to independently predict the risk for WNND-related death. The association between age and severe disease has been reported (9-11). The contributing factor of age may relate to a decrease in the integrity of the blood-brain barrier and facilitate access of WNV to the central nervous system (13). Heart disease, particularly cardiac arrhythmias, have also been recognized as frequent contributors to death caused by WNV encephalitis (13-15). Physiologic stress of WNV infection may precipitate or exacerbate underlying medical conditions resulting in death (14).

These findings emphasize the need for primary prevention of WNV infection in patients with these predisposing conditions and close monitoring for cardiac complications in elderly patients hospitalized with WNV disease. Vector mosquito control programs, including source reduction and larviciding of Culex spp. mosquitoes and ongoing public health education and WNV surveillance in disease-endemic and newly affected areas, remain the cornerstones of WNV disease prevention and control.

\section{Acknowledgments}

We thank the physicians at the hospitals and local public health authorities for providing assistance with surveillance of WNV infections in Greece; personnel in the Reference 
West Nile Virus Infection in Greece, 2010

Table 2. Predictive factors of death for 197 patients with West Nile neuroinvasive disease analyzed by univariate and multivariate analysis, Greece, July-October 2010*

\begin{tabular}{|c|c|c|c|c|}
\hline Characteristic & No. deaths, $n=33 \dagger$ & Case-fatality rate, $\%$ & Crude risk ratio $(95 \% \mathrm{Cl})$ & Adjusted risk ratio $\neq(95 \% \mathrm{Cl})$ \\
\hline \multicolumn{5}{|l|}{ Age group, y } \\
\hline $40-59$ & $1 \S$ & 2.50 & Reference & Reference \\
\hline $60-69$ & 2 & 6.90 & $2.76(0.26-28.99)$ & $2.72(0.26-28.40)$ \\
\hline $70-79$ & 15 & 17.65 & $7.06(0.97-51.59)$ & $6.13(0.83-45.17)$ \\
\hline$\geq 80$ & 15 & 34.88 & $13.95(1.93-100.9)$ & $11.41(1.56-83.52)$ \\
\hline \multicolumn{5}{|l|}{ Sex } \\
\hline $\mathrm{F}$ & 10 & 11.36 & Reference & NA \\
\hline M & 23 & 21.10 & $1.86(0.93-3.69)$ & NA \\
\hline \multicolumn{5}{|c|}{ Underlying diseases } \\
\hline None & 2 & 3.92 & Reference & NA \\
\hline$\geq 1$ & 31 & 21.23 & $5.41(1.34-21.82)$ & NA \\
\hline \multicolumn{5}{|l|}{ Hypertension } \\
\hline No & 19 & 15.70 & Reference & NA \\
\hline Yes & 14 & 18.42 & $1.17(0.63-2.20)$ & NA \\
\hline \multicolumn{5}{|l|}{ Heart disease } \\
\hline No & 18 & 12.00 & Reference & Reference \\
\hline Yes & 15 & 32.61 & $2.72(1.49-4.95)$ & $2.03(1.14-3.64)$ \\
\hline \multicolumn{5}{|l|}{ Diabetes } \\
\hline No & 23 & 15.44 & Reference & NA \\
\hline Yes & 10 & 20.83 & $1.35(0.69-2.63)$ & NA \\
\hline \multicolumn{5}{|c|}{ Immunosuppression } \\
\hline No & 31 & 17.42 & Reference & NA \\
\hline Yes & 2 & 10.53 & $0.60(0.16-2.33)$ & NA \\
\hline \multicolumn{5}{|l|}{ Cancer } \\
\hline No & 29 & 16.11 & Reference & NA \\
\hline Yes & 4 & 23.53 & $1.46(0.58-3.66)$ & NA \\
\hline \multicolumn{5}{|l|}{ Stroke } \\
\hline No & 28 & 15.30 & Reference & NA \\
\hline Yes & 5 & 35.71 & $2.33(1.07-5.10)$ & NA \\
\hline \multicolumn{5}{|l|}{ Renal failure } \\
\hline No & 31 & 16.40 & Reference & NA \\
\hline Yes & 2 & 25.00 & $1.52(0.44-5.28)$ & NA \\
\hline
\end{tabular}

${ }^{*} \mathrm{Cl}$, confidence interval; NA, not applicable.

†Two additional patients with nonneuroinvasive disease died, and those deaths were not included in this analysis.

In logistic regression analysis, initial models included all variables for which the $p$ value was $<0.05$ or the odds ratio was $>1.1$ or $<0.90$. Therefore, all variables were included in the initial models. Variables were removed 1 at a time depending on results of statistical testing ( $p<0.05$ ), by using the likelihood-ratio test. All variables that remained significant in the final logistic regression model were included in the binomial regression model for the estimation of adjusted risk ratios.

§Belonged to the 40-49-year age group.

Laboratory for Arboviruses for providing technical support; personnel of HCDCP and the outbreak coordinating team for organizing and implementing prevention and control program; scientists and staff of Ecodevelopment SA for contributing to mosquito control operations; and V. Sambri for performing neutralization testing.

This study was supported by the HCDCP.

Dr Danis is a medical epidemiologist at the Office of Vectorborne Diseases and Zoonoses, Department of Surveillance, Hellenic Centre for Disease Control and Prevention, Athens, Greece. His research interests include epidemiology of vectorborne and vaccine-preventable diseases and surveillance of communicable diseases.

\section{References}

1. Papa A, Danis K, Baka A, Bakas A, Dougas G, Lytras T, et al. Ongoing outbreak of West Nile virus infections in humans in Greece, July-August 2010. Euro Surveill. 2010;15:pii:19644.

2. Papa A, Xanthopoulou K, Gewehr S, Mourelatos S. Detection of West Nile virus lineage 2 in mosquitoes during a human outbreak in Greece. Clin Microbiol Infect. 2011;17:1176-80. doi:10.1111/ j.1469-0691.2010.03438.x

3. Pavlatos M, Smith CE. Antibodies to arthropod-borne viruses in Greece. Trans R Soc Trop Med Hyg. 1964;58:422-4. doi:10.1016/0035-9203(64)90089-6

4. Antoniadis A, Alexiou-Daniel S, Malisiovas N, Doutsos I, Polyzoni T, Leduc JW, et al. Seroepidemiological survey for antibodies to arboviruses in Greece. Arch Virol. 1990;Suppl 1:277-85.

5. Papa A, Perperidou P, Tzouli A, Castiletti C. West Nile virus-neutralizing antibodies in humans in Greece. Vector Borne Zoonotic Dis. 2010;10:655-8. doi:10.1089/vbz.2010.0042 
6. Kantzanou MN, Moschidis ZM, Kremastinou G, Levidiotou S, Karafoulidou A, Politis C, et al. Searching for West Nile virus (WNV) in Greece. Transfus Med. 2010;20:113-7. doi:10.1111/ j.1365-3148.2009.00964.x

7. Papa A, Karabaxoglou D, Kansouzidou A. Acute West Nile virus neuroinvasive infections: cross-reactivity with dengue virus and tick-borne encephalitis virus. J Med Virol. 2011. In press.

8. European Centre for Disease Control and Prevention (ECDC). West Nile virus transmission in Europe [cited 2010 Sep 10]. http://ecdc. europa.eu/en/activities/sciadvice/Lists/ECDC\%20Reviews/ECDC_ DispForm.aspx? List $=512 \mathrm{ff} 74 \mathrm{f}-77 \mathrm{~d} 4-4 \mathrm{ad} 8$-b6d6-bf0f23083f30\&ID $=940 \&$ RootFolder $=\% 2 \mathrm{Fen} \% 2 \mathrm{Factivities} \% 2 \mathrm{Fsciadvice} \% 2 \mathrm{FLists} \%$ 2FECDC\%20Reviews

9. Tsai TF, Popovici F, Cernescu C, Campbell GL, Nedelcu NI. West Nile encephalitis epidemic in southeastern Romania. Lancet. 1998;352:767-71. doi:10.1016/S0140-6736(98)03538-7

10. Lindsey NP, Staples JE, Lehman JA, Fischer M; Centers for Disease Control and Prevention. Surveillance for human West Nile virus disease-United States, 1999-2008. MMWR Surveill Summ. 2010;59:1-17.

11. Weinberger M, Pitlik SD, Gandacu D, Lang R, Nassar F, Ben David D, et al. West Nile fever outbreak, Israel, 2000: epidemiologic aspects. Emerg Infect Dis. 2001;7:686-91. doi:10.3201/ eid0704.010416
12. Papa A, Bakonyi T, Xanthopoulou K, Vasquez A, Tenorio A, Nowotny N. Genetic characterization of a neuroinvasive lineage 2 West Nile virus, Greece, 2010. Emerg Infect Dis. 2011;17:920-2.

13. Weiss D, Carr D, Kellachan J, Tan C, Phillips M, Bresnitz E, et al. Clinical findings of West Nile virus infection in hospitalized patients, New York and New Jersey, 2000. Emerg Infect Dis. 2001;7:654-8. doi:10.3201/eid0704.010409

14. Sejvar JJ, Lindsey NP, Campbell GL. Primary causes of death in reported cases of fatal West Nile Fever, United States, 2002-2006. Vector Borne Zoonotic Dis. 2011;11:161-4. doi:10.1089/vbz.2009.0086

15. Sejvar JJ. The long-term outcomes of human West Nile virus infection. Clin Infect Dis. 2007;44:1617-24. doi:10.1086/518281

Address for correspondence: Kostas Danis, Department of Surveillance and Intervention, Hellenic Centre for Disease Prevention and Control, Agrafon 3-5, Marousi, Athens 15123, Greece; email: daniscostas@yahoo. com

The opinions expressed by authors contributing to this journal do not necessarily reflect the opinions of the Centers for Disease Control and Prevention or the institutions with which the authors are affiliated.

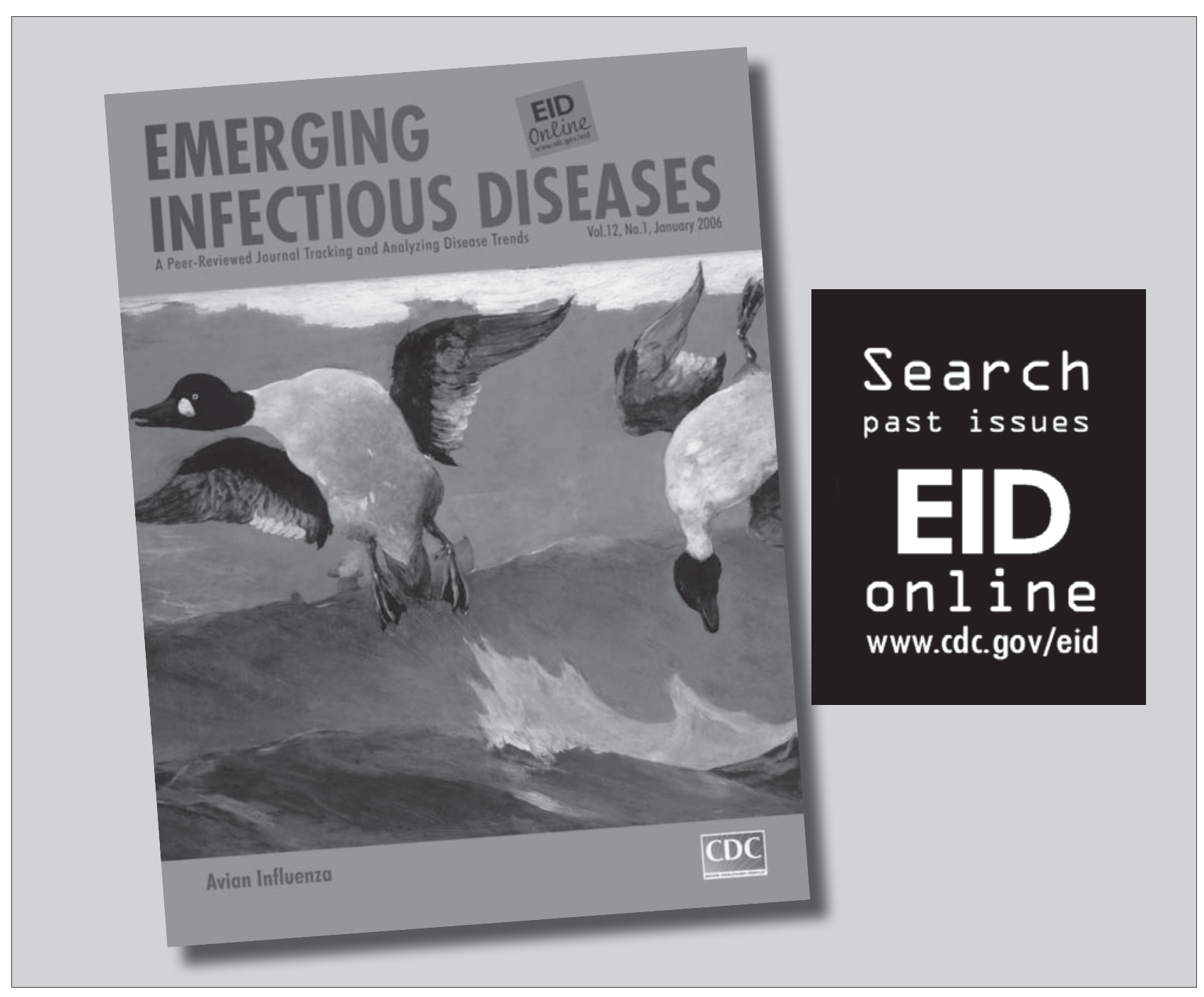

